\title{
Physiotherapist Involvement in Concussion Services in New Zealand: A National Survey
}

Sophie Maxtone BPhty(Hons)

Centre for Health, Activity and Rehabilitation Research, School of Physiotherapy, University of Otago, Dunedin, New Zealand

Megan Bishop MHSC

Centre for Health, Activity and Rehabilitation Research, School of Physiotherapy, University of Otago, Dunedin, New Zealand

Cathy Chapple $P h D$

Centre for Health, Activity and Rehabilitation Research, School of Physiotherapy, University of Otago, Dunedin, New Zealand

Steve Tumilty PhD

Centre for Health, Activity and Rehabilitation Research, School of Physiotherapy, University of Otago, Dunedin, New Zealand

Dusty Quinn MMpty

Back In Motion, Dunedin, New Zealand

Ewan Kennedy $P h D$

Centre for Health, Activity and Rehabilitation Research, School of Physiotherapy, University of Otago, Dunedin, New Zealand

\section{ABSTRACT}

The purpose of this study was to describe physiotherapist involvement in concussion services in New Zealand. This would enable a comparison with international recommendations for concussion care, and evaluation of physiotherapy concussion care in New Zealand to help determine what is successful and what could be improved. The study involved a national online survey of physiotherapists distributed via Physiotherapy New Zealand (PNZ) branches and special interest groups. The responses of 175 participants were analysed, representing approximately $5 \%$ of PNZ members. Respondents were commonly involved in the recognition $(107 ; 61 \%)$, assessment $(133 ; 76 \%)$, and management $(154 ; 88 \%)$ of concussion in a wide range of primary care concussion services in various settings/contexts and under different funding schemes. Respondents reported frequently assessing and managing disorders in the physiological brain, vestibulo-ocular, and cervicogenic sub-systems. Overall, physiotherapists currently provide a wide range of primary care services for people with concussion that aligns with international recommendations, especially in early active rehabilitation and screening for concurrent injuries. Key challenges highlighted by this research include people presenting late to physiotherapy, continuity of care, and the frequency of persistent or recurrent symptoms.

\section{Maxtone, S., Bishop, M., Chapple, C., Tumilty, S., Quinn, D., \& Kennedy, E. (2020). Physiotherapist involvement in concussion services in New Zealand: A national survey. New Zealand Journal of Physiotherapy, 48(2), 70-79. https://doi. org/10.15619/NZJP/48.2.03}

Key Words: Brain Concussion, Physiotherapy, Rehabilitation, Healthcare

\section{INTRODUCTION}

Concussion is a complex problem. While widely defined as a form of mild traumatic brain injury (McCrory et al., 2017), the potential for concurrent cervical spine, vestibular, and oculomotor injuries is increasingly recognised (Elkin et al., 2016; Ellis et al., 2015; Leslie \& Craton, 2013; Schneider et al., 2014; van der Walt et al., 2019). Reflecting this evolving understanding, current best practice in concussion care involves a review of multiple systems followed by an active approach to rehabilitation, and often multiple professions (Schneider, 2019a, 2019b). Concussion can be classified into physiological (brain), vestibulo-ocular, and cervicogenic post-concussion disorders (Ellis et al., 2015). This approach recognises the heterogeneous nature of concussion, and encourages evaluation and management of impairments in each subsystem. The traditional model of rest is increasingly recognised as unhelpful beyond the first 24-48 hours following injury (McCrory et al., 2017; Schneider et al., 2013), with an active approach to recovery increasingly recommended (Leddy et al., 2016; Leddy et al., 2019; Willer et al., 2019). These developments present challenges for primary care concussion services, but also an opportunity for physiotherapists to take a greater role in people's recovery.

Physiotherapists are well positioned to contribute to concussion care, with a diverse and unique skill set in active rehabilitation as well as the evaluation and management of cervical spine and vestibulo-ocular disorders (Schneider, 2019a). In New Zealand, physiotherapists are well-established primary healthcare providers, with direct access to services without a referral. Physiotherapists are present in sporting contexts and in the community where concussion injuries are commonly sustained, are well-established providers of rehabilitation for people with neurological conditions, and members of multidisciplinary concussion services. Recent work indicates that within a multidisciplinary concussion service, physiotherapy treatment of cervical spine and vestibulo-ocular issues was recommended 
in $86 \%$ of cases (van der Walt et al., 2019). These data exclude the key worker role, which is often also performed by physiotherapists, so could under-represent physiotherapy involvement. This highlights the large contribution physiotherapists could make to concussion rehabilitation. The extent to which physiotherapists are involved in concussion care outside this multidisciplinary service is less clear.

Providers of concussion services must be able to explore a range of potential symptom sources and provide active individualised rehabilitation. While physiotherapists are well positioned to contribute to concussion care, the extent to which they are currently involved is not clear. In order to benefit from the considerable potential of physiotherapists to contribute to people's recovery from concussion, a better understanding of physiotherapist involvement is necessary. This understanding must encompass a wide range of potential involvement in concussion recognition, assessment, and management under a range of health services. In New Zealand, many concussion services are partially or fully funded by the Accident Compensation Corporation (ACC), a national funder of accident-related injuries, including concussion. The aim of this study was to understand physiotherapist involvement in the recognition, assessment, and management of concussion in New Zealand. This would enable a comparison between current practice and international recommendations, evaluation of areas of success, and identification of areas that could be improved.

\section{METHODS}

This study involved a cross-sectional online survey completed by New Zealand physiotherapists with a current annual practicing certificate involved in the care of people with concussion. Ethics approval was granted by the University of Otago Human Ethics Committee (D19/187).

\section{Survey development}

The online survey was created using specialised survey software (Qualtrics ${ }^{\mathrm{XM}}$ ), available via the University of Otago. Survey questions were developed by the research team and organised into a series of six "blocks": survey information and consent, demographics, recognition, assessment, management, and continuity of care. Survey flow logic was utilised so that respondents would be directed to answer questions within the blocks relevant to their involvement in concussion care. This would reduce the survey time for those with lower levels of involvement in concussion care.

The demographic block was aligned with the workforce survey conducted annually by the Physiotherapy Board of New Zealand (Physiotherapy Board of New Zealand, 2018). It included questions regarding the experience of physiotherapists involved in concussion care and their work characteristics. Respondents were then asked if they were involved in the recognition, assessment, and/or management of concussion, and based on this, directed to other relevant questions. "Recognition" was defined as "the identification of an individual with suspected concussion". This block included questions about the context of recognition, the tools and skills used when recognising concussion, and the subsequent care of people with a recognised concussion. "Assessment" was defined as "the evaluation of an individual with suspected/confirmed concussion". This block included questions about how physiotherapy services are accessed and funded; what sub-systems are being assessed; the timeframe for initial presentation for assessment, referral and other aspects of subsequent management; and the context/setting of assessment. The management block included questions about the setting up and funding of services, the sub-systems managed by the service, the frequency of people returning with persistent problems, and the typical number and timeframe of appointments. All respondents then concluded the survey by completing the "continuity of care" block. Survey questions were typically multiple choice, with "other" responses available. Selected questions requested an open text response.

The survey questions and flow were developed in an iterative process, beginning with development and trials within the research team, followed by a peer-review process and then trials with a small number of local physiotherapists. Each iteration improved the clarity and flow of the questions and the survey design.

\section{Survey distribution}

This research involved a national survey of a cross-section of registered physiotherapists involved in the recognition, assessment, and/or management of concussion in New Zealand. The link to the online survey was distributed electronically via email, social media, websites, and other online platforms via professional physiotherapy networks, including Physiotherapy New Zealand (PNZ) branches and special interest groups, and professional contacts of the research group.

Invitations to participate in the survey were distributed in June 2019 and remained open for an 8-week period (June-August 2019). Access to the survey was via an anonymous electronic link; those that were interested in participating were directed to an information sheet at the start of the survey. Participation was voluntary and responses were self-reported.

Data extraction and analysis

The data set was exported from Qualtrics ${ }^{\mathrm{XM}}$ to Microsoft Excel, and was limited to survey responses collected during the 8-week period. Responses from those who declined to proceed or that were evidently incomplete (e.g. only the first few questions were answered) were excluded from the data set. The remaining responses were formatted and transferred to IBM SPSS $\mathbb{R}$ Statistics 25 for analysis. The data analysis primarily involved descriptive statistics utilising IBM SPSS Statistics. The open text responses were analysed with a conventional content analysis (Hsieh \& Shannon, 2005) by consensus between two members of the research team (SM and EK). Responses were categorised and described with minimal abstraction (Vaismoradi et al., 2016), consistent with the level of content in the relatively short text responses. Consideration of wider themes based on all the quantitative and qualitative results framed the discussion.

\section{RESULTS}

\section{Response rate}

Responses to the survey are shown in Figure 1. Of the 3,538 PNZ members at the time of the survey (Physiotherapy New Zealand, 2019), 175 completed the survey in full, representing approximately $5 \%$ of PNZ members. 
Figure 1

Survey Responses

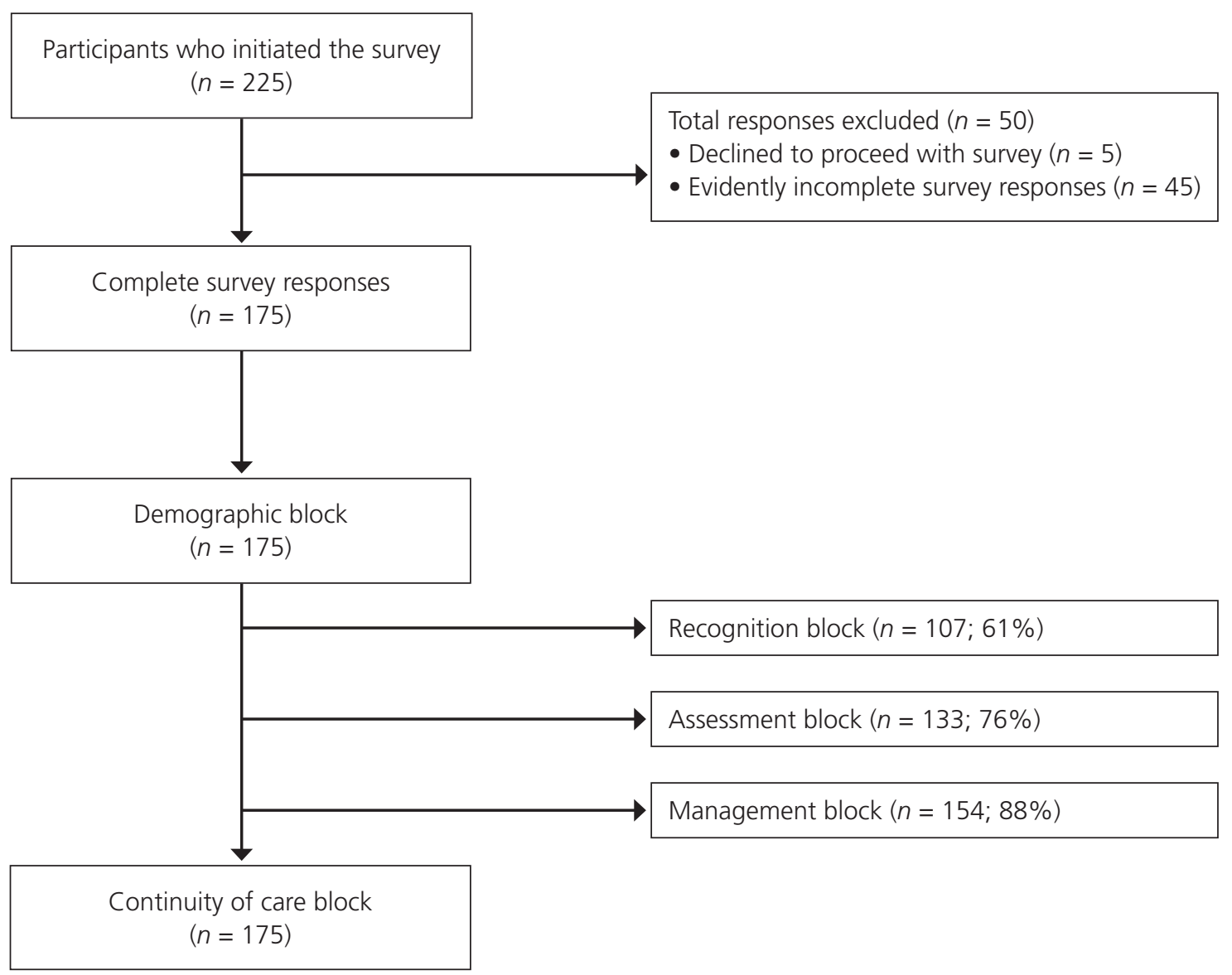

Demographics, work characteristics, and involvement in concussion care

The demographic and work characteristics of respondents are provided in Table 1. Of the respondents, physiotherapists were most commonly involved in the management of concussion (154; 88.0\%), followed by assessment (133; 76.0\%), and recognition $(107 ; 61.1 \%)$, as outlined in Table 2 . A majority of respondents $(140 ; 80 \%)$ were involved in more than one area of concussion care, with $43(24.6 \%)$ involved in assessment and management, and 77 (44.0\%) involved in recognition, assessment, and management.

\section{Recognition of concussion}

The contexts in which respondents were involved in the recognition of concussion were primary care $(71 ; 66.4 \%)$, affiliation to a sports team or athlete $(62 ; 57.9 \%)$, or less commonly, an acute setting (15; 14.0\%). "Other" text responses $(6 ; 5.6 \%)$ indicated that recognition also occurred within the ACC concussion service.

\section{Table 1}

Respondent Characteristics

\begin{tabular}{lc}
\hline Characteristic & Frequency $(\%)$ \\
\hline Gender & \\
Male & $49(28)$ \\
Female & $126(72)$ \\
Age bracket (years) & \\
$20-34$ & $64(37)$ \\
$35-44$ & $59(34)$ \\
$45-54$ & $37(21)$ \\
$55-64$ & $14(8)$ \\
$65+$ & $1(1)$ \\
\hline
\end{tabular}




\begin{tabular}{|c|c|}
\hline Characteristic & Frequency (\%) \\
\hline \multicolumn{2}{|l|}{ Ethnicity } \\
\hline New Zealand European & $133(76)$ \\
\hline Māori & $8(5)$ \\
\hline Chinese & $8(5)$ \\
\hline Indian & $4(2)$ \\
\hline Other & $32(18)$ \\
\hline \multicolumn{2}{|l|}{ Highest qualification } \\
\hline Diploma or graduate diploma & $12(7)$ \\
\hline Bachelor's degree & $70(40)$ \\
\hline Postgraduate diploma or certificate & $62(35)$ \\
\hline Master's degree & $28(16)$ \\
\hline Doctoral degree & $3(2)$ \\
\hline \multicolumn{2}{|l|}{ District Health Board region a } \\
\hline Auckland & $20(12)$ \\
\hline Counties Manukau & $12(7)$ \\
\hline Waikato & $26(15)$ \\
\hline Bay of Plenty & $16(9)$ \\
\hline Capital and Coast & $10(6)$ \\
\hline Other, North Island b & $28(16)$ \\
\hline Canterbury & $31(18)$ \\
\hline Southern & $25(15)$ \\
\hline Other, South Island b & $3(2)$ \\
\hline \multicolumn{2}{|c|}{ Experience working with people with concussion (years) } \\
\hline $1-3$ & $67(38)$ \\
\hline $4-6$ & $43(25)$ \\
\hline $7-9$ & $9(5)$ \\
\hline $10+$ & $56(32)$ \\
\hline \multicolumn{2}{|c|}{ Time per week working with people with concussion (hours) } \\
\hline $1-10$ & $151(86)$ \\
\hline $11-30$ & $22(13)$ \\
\hline $31+$ & $2(1)$ \\
\hline \multicolumn{2}{|c|}{ Main area of practice working with people with concussion } \\
\hline Musculoskeletal outpatients & $54(31)$ \\
\hline Sports physiotherapy & $60(34)$ \\
\hline Adult neurology & $26(15)$ \\
\hline Community/domiciliary & $10(6)$ \\
\hline Occupational health & $6(3)$ \\
\hline Other ${ }^{c}$ & $25(14)$ \\
\hline
\end{tabular}

Note. $N=175$.

a Three responses missing. ${ }^{b}$ Areas with $<5 \%$ of respondents (Hawke's Bay, Hutt Valley, Lakes, MidCentral, Nelson Marlborough, Northland, South Canterbury, Taranaki, Wairarapa, Waitematā, West Coast, Whanganui). c Areas with $<3 \%$ of respondents (cardiovascular/pulmonary inpatient, cardiovascular/pulmonary outpatient, continuing care, mental health, management, older adult, other paediatric, oncology, paediatric neurology).

\section{Table 2}

How People With Concussion Most Commonly Access Physiotherapy Services

\begin{tabular}{lc}
\hline Method of access & Frequency (\%) \\
\hline Referral directly from ACC or ACC concussion service provider & $60(45)$ \\
Referral by medical practitioner following concussion diagnosis & $21(16)$ \\
Person self-refers for concussion-specific problems & $19(14)$ \\
Person self-refers for other problems, but upon assessment, physiotherapist suspects concussion & $16(12)$ \\
Other & $17(13)$ \\
\hline
\end{tabular}

Note. $N=133 . A C C=$ Accident Compensation Corporation . 
Clinical judgement $(80 ; 74.8 \%)$ and the Sports Concussion Assessment Tool $(76 ; 71.0 \%)$ were commonly utilised in the recognition of concussion. "Other" tools and skills specified more than once in text responses included Vestibular-ocular Motor Screening (VOMS), the Rivermead Post-Concussion Symptom Questionnaire, and the Buffalo Concussion Treadmill Test.

A large proportion of respondents indicated that, following recognition, they were involved in that person's subsequent care $(93 ; 86.9 \%)$. When prompted to describe how they were involved, respondents described referral to general practictioners (GPs), concussion services or other physiotherapists; monitoring return to play/sport/work/school; cervical spine treatment; and vestibulo-ocular rehabilitation. Those who reported not being involved in subsequent care following recognition (14; 13.1\%) commonly referred people to their GP or concussion services for subsequent care.

\section{Assessment of concussion}

Table 2 presents the way people with concussion most commonly accessed physiotherapy services for assessment. "Other" responses (17; 12.8\%) described access via a sports team or other sporting environment. The setting in which physiotherapy assessment was provided is shown in Table 3.

The funding scheme(s) respondents used to provide concussion assessment and the respective sub-system are shown in Figure 2. Comparatively, participants most commonly performed vestibulo-ocular assessment under the ACC concussion service (81\%), and cervical spine or other musculoskeletal assessment under a fee-for-service scheme (96\%). Less than half of the

\section{Table 3}

Settings Where Physiotherapy Services for Concussion Assessment and/or Management are Provided

\begin{tabular}{lcc}
\hline Setting & \multicolumn{2}{c}{ Frequency (\%) } \\
\cline { 2 - 3 } & $\begin{array}{c}\text { Assessment } \\
(n=133)\end{array}$ & $\begin{array}{c}\text { Management } \\
(n=119)^{\text {a }}\end{array}$ \\
\hline Person's home and/or their local community & $37(28)$ & $36(30)$ \\
Clinic setting & $108(81)$ & $100(84)$ \\
Hospital setting & $5(4)$ & $3(3)$ \\
Sporting grounds or facilities & $48(36)$ & $28(24)$ \\
Other & $2(2)$ & $4(3)$ \\
\hline
\end{tabular}

Note. Respondents could select more than one answer.

35 responses missing.

\section{Figure 2}

Funding Scheme for Physiotherapy Assessment of Concussion and Respective Sub-System Assessed

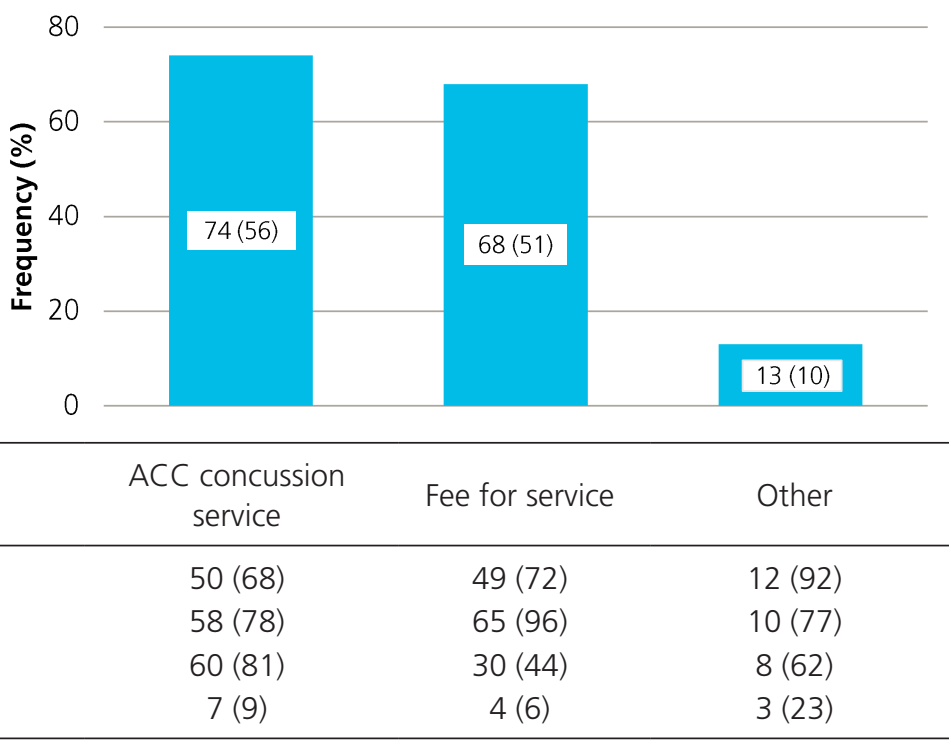

\begin{tabular}{lccc}
\hline & ACC concussion & Fee for service & Other \\
& service & $49(72)$ & $12(92)$ \\
Physiological brain injury & $50(68)$ & $65(96)$ & $10(77)$ \\
Cervical spine or other musculoskeletal dysfunction & $58(78)$ & $30(44)$ & $8(62)$ \\
Vestibulo-ocular system & $60(81)$ & $7(9)$ & $3(23)$ \\
Other & $7(6)$ & 4 \\
\hline
\end{tabular}

Note. $N=133$. Data are frequency (\%). Respondents could select more than one answer. The left column outlines the respective sub-systems. $\mathrm{ACC}=$ Accident Compensation Corporation. 
respondents typically conducted their initial assessment within 1 week of the sustained concussion (50; 37.6\%), with other respondents conducting this assessment within 2 weeks (22; $16.5 \%)$, within 4 weeks $(36 ; 27.1 \%)$ or beyond 4 weeks $(25$ : $18.8 \%)$.

The majority of respondents $(115 ; 86.5 \%)$ were involved in the subsequent management of a person's concussion following assessment. The $18(13.5 \%)$ respondents not typically involved in subsequent management, most commonly referred people to a GP or concussion services/clinic for subsequent management.

\section{Management of concussion}

The funding scheme(s) respondents accessed to provide concussion management and the respective sub-system being managed is provided in Figure 3. "Other" responses (14; 9.1\%) included providing services under funding from sports teams/ organisations, non-ACC private physiotherapy or from a hospital setting. Aspects of concussion management frequently specified in "other" text entry responses included sleep hygiene and rehabilitation for functional independence.
A majority of respondents (120; 77.9\%) involved in the management of concussion reported that people returned with persistent problems (Table 4). The nature of these problems is presented in Figure 4, with "other" text responses specifying repeat concussion injury, and ongoing difficulty with memory, fatigue, and concentration as reason for returning for further treatment.

The settings in which respondents provided concussion management services are presented in Table 3. The typical number and timespan of appointments prior to discharge are presented in Table 5. During concussion management, 67.3\% (103) of respondents reported people did not attend follow-up appointments at least "sometimes".

\section{Continuity of care}

Over half of respondents $(112 ; 65.5 \%)$ reported that the same health professionals involved in the recognition and assessment of a person's concussion are typically involved in the management of that person's concussion, while 59 (34.5\%) reported that this was not the case. Respondents who indicated

\section{Figure 3}

Funding Scheme for Physiotherapy Management of Concussion and Respective Sub-System Managed

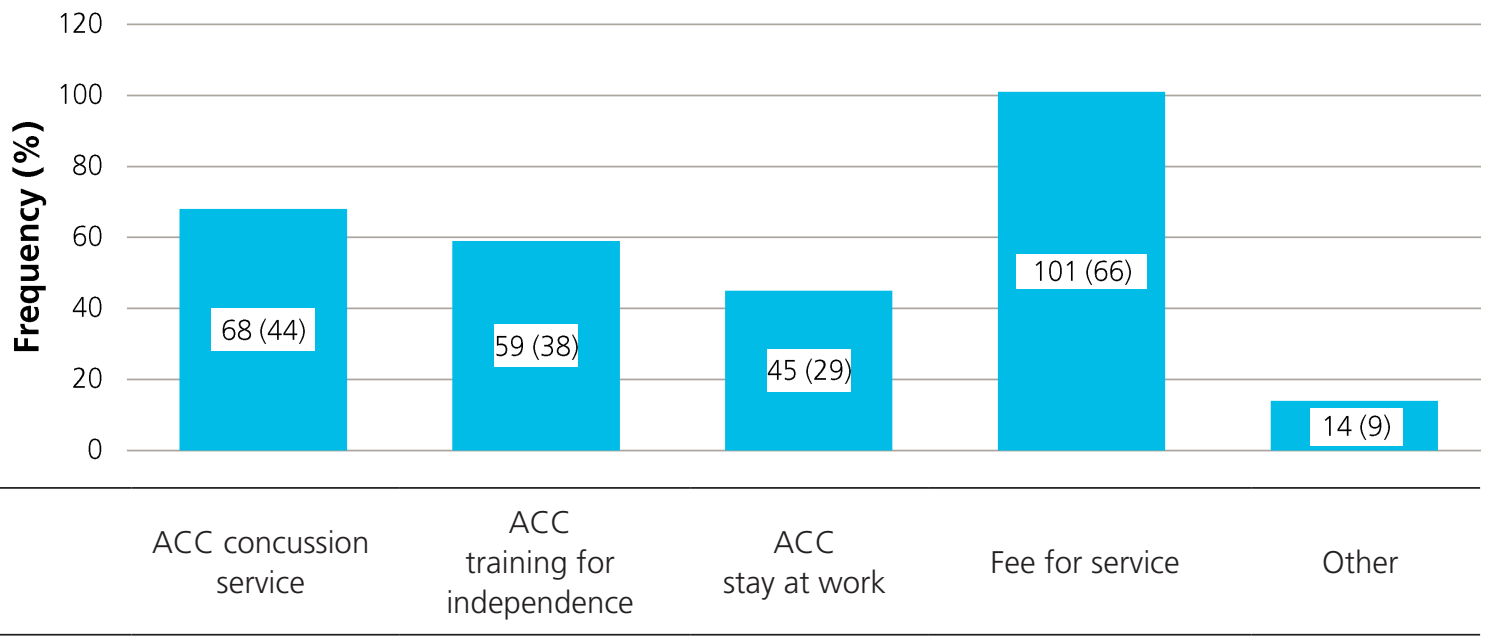

Physiological brain injury

Education

$58(85)$

$51(86)$

$35(78)$

$87(86)$

$13(93)$

Progressive aerobic exercise

$56(82)$

51 (86)

$34(76)$

$70(69)$

$11(85)$

Graduated return to sport/

$47(69)$

$46(80)$

$42(93)$

$73(72)$

$11(85)$

school/work

Other

$7(10)$

$4(7)$

$3(7)$

$4(4)$

$1(8)$

Cervical spine

Cervical spine management

$50(74)$

38 (64)

29 (64)

$95(94)$

11 (85)

Other

6 (9)

$3(5)$

$1(2)$

1 (1)

$1(8)$

Vestibulo-ocular system

Vestibular rehabilitation

$56(82)$

$44(75)$

$18(40)$

$40(40)$

$7(54)$

Oculomotor rehabilitation

$54(79)$

$42(71)$

$15(33)$

$30(30)$

$6(46)$

Balance retraining

$50(85)$

$22(49)$

$60(59)$

$10(77)$

$5(7)$

$3(5)$

$1(2)$

$1(1)$

$1(8)$

Note. $N=154$. Data are frequency (\%). Respondents could select more than one answer. The left column outlines the respective sub-systems and management. $\mathrm{ACC}=$ Accident Compensation Corporation . 


\section{Table 4}

Frequency People Return for Further Management due to Persistent Problems Related to Their Concussion, Following Discharge From Physiotherapy Services

\begin{tabular}{lr}
\hline Frequency of return & Frequency (\%) \\
\hline Always & $3(2)$ \\
Most of the time & $2(1)$ \\
About half the time & $4(3)$ \\
Sometimes & $111(72)$ \\
Never & $34(22)$ \\
\hline
\end{tabular}

Note. $N=154$

\section{Figure 4}

Persistent Problems of Those Returning for Further Management of Concussion Following Initial Discharge

80

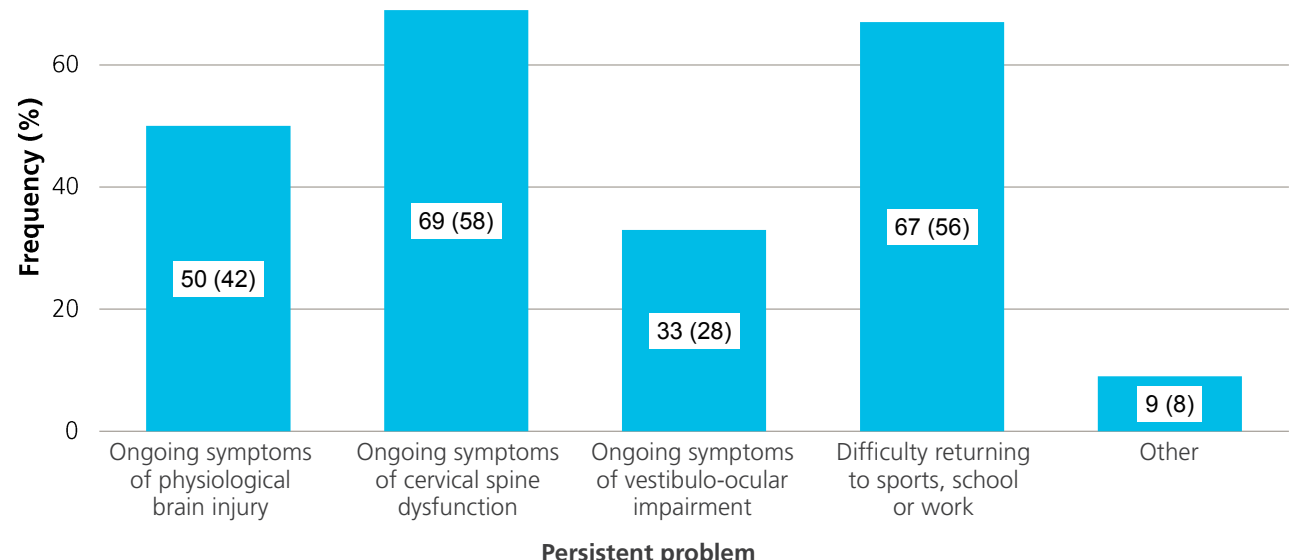

Note. $N=120$. Respondents who indicated that people "never" returned with persistent symptoms (as shown in Table 4) were not displayed in this figure.

\section{Table 5}

Number and Timespan of Appointments Prior to Discharge

\begin{tabular}{lc}
\hline Appointments & Frequency $(\%)$ \\
\hline Typical number, prior to discharge $(n=152)^{\text {a }}$ & $36(24)$ \\
$1-4$ & $100(66)$ \\
$5-9$ & $16(11)$ \\
$10+$ & $23(15)$ \\
Typical timespan for reschedule $(n=151)^{b}$ & $22(15)$ \\
Within 1 week & $39(26)$ \\
Within 2 weeks & $67(44)$ \\
Within 4 weeks & \\
$>4$ weeks & \\
\hline
\end{tabular}

Note. $N=154$.

${ }^{a}$ Two missing responses. ${ }^{b}$ Three missing responses. 
that this was not the case were asked to further describe how people transitioned between health professionals. Responses described the need for people to access multiple services (such as medical care and the concussion service) and care from multiple health professionals with relevant expertise (such as GPs, occupational therapists, and physiotherapists with expertise in the cervical spine or vestibulo-ocular system). Numerous transitions between health professionals to receive concussion care were described.

In response to the question "Are you involved in the prevention of concussion?", 121 participants (70\%, 3 missing responses) answered "no". Those who answered "yes" (51; 30\%) were most commonly involved in providing education that addressed concussion risks to coaches, sporting teams or groups, parents, GPs, and schools. Other forms of involvement included neck strengthening, addressing sporting technique such as in tackling, and advising on protective equipment.

\section{DISCUSSION}

This study aims to contribute to a better understanding of New Zealand physiotherapist involvement in concussion recognition, assessment, and management. Respondent demographics are comparable with the demographics of New Zealand physiotherapists as reported by the Physiotherapy Board of New Zealand (Physiotherapy Board of New Zealand, 2018), encompassing a wide range of regions, areas of practice, and levels of experience. These data would enable a discussion about how New Zealand physiotherapists contribute to concussion services, if their approach reflects international best practice in concussion care, and whether current services could be improved.

The study's findings highlight that physiotherapists in New Zealand are involved in a wide range of services for people with concussion. Physiotherapy involvement encompasses a variety of purposes (recognition, assessment, and management), settings, stages post injury, types of concussion services, areas of assessment, and management. It is clear that physiotherapists have a far more complex role than the recognition of concussion and referral to a medical doctor, as is implied in some publications (Accident Compensation Corporation, 2016). Illustrating this point, $80 \%$ of respondents were involved in more than one area of recognition, assessment or management of concussion. Most of those involved in the recognition of concussion (87\%) were typically involved in that person's subsequent care, going on to provide further assessment and management as well as onward referral as appropriate. In other words, just $13 \%$ of those involved in recognition of concussion described their subsequent involvement as limited to onward referral. Responses were consistent with an early active approach to concussion care, which is now widely recommended (Leddy et al., 2016; Leddy et al., 2018; Leddy et al., 2019; Marshall et al., 2015; McCrory et al., 2017; Reneker et al., 2017; Schneider et al., 2014; Schneider, 2019a, 2019b; Willer et al., 2019). However, this does not mean that physiotherapists are commonly or consistently involved in concussion services. The low overall response rate suggests that although those who are involved in concussion services contribute to a wide range of services, more work is needed to engage a greater number of physiotherapists in the care of people with concussion. Further education and awareness of concussion within the profession is suggested.

Physiotherapists have a diverse skill set that facilitates assessment and management of key sub-systems affected in concussion injuries. Ellis et al. (2015) describe three postconcussion disorders based on the system primarily affected: physiological (brain), cervicogenic, and vestibulo-ocular. Treatment for these disorders is described as an early active sub-symptom threshold exercise for physiological, and targeted neck and vestibulo-ocular rehabilitation. As illustrated in Figures 2-4, physiotherapists report commonly addressing each of these aspects in concussion assessment and management across a variety of services. This highlights the ability of physiotherapists to provide comprehensive concussion assessment and targeted management that aligns with the complex nature of concussion recovery (Schneider, 2019a, 2019b). The management of disturbance to brain physiology as a result of concussion can be addressed with education, progressive aerobic exercise, and graduated return to school/sport/work (Ellis et al., 2015; Leddy et al., 2012; Leddy \& Willer, 2013; McCrory et al., 2017). Neck and vestibulo-ocular issues are prevalent in people with persistent symptoms post-concussion (van der Walt et al., 2019), and it is widely accepted that concussion assessment and management should address these systems (Ellis et al., 2015; McCrory et al., 2017; Schneider et al., 2014; Schneider, 2019b), particularly in those with persistent symptoms (Kennedy et al., 2019; Leddy et al., 2012; Leddy et al., 2016; Schneider, 2019a). Low reported involvement in the prevention of concussion (30\%) likely reflects a lack of current evidence for proposed strategies (Schneider et al., 2017). Those who did report involvement described strategies consistent with reported literature (Schneider et al., 2017), with an emphasis on education. While this educational approach may lack evidence for primary prevention, it undoubtedly plays a useful role in secondary prevention - reducing the impact of concussion injuries through effective evaluation and management.

The findings highlight several challenges in the provision of concussion services. For nearly half of respondents (45.9\%), the initial assessment of a person presenting with symptoms of concussion was conducted later than 2 weeks after the suspected concussion was sustained. At this stage symptoms are considered to be persistent (McCrory et al., 2017), and may warrant a more comprehensive multidisciplinary assessment (Schneider, 2019a). Medical referral for formal confirmation of the diagnosis could further delay physiotherapy care. However, people appear to commonly present to physiotherapy without a medical diagnosis of concussion (up to 39\%; see Table 2). This late presentation to physiotherapy may negatively affect people's outcomes, as current evidence indicates that rest beyond 24-48 hours of the injury may lead to poorer outcomes (Leddy et al., 2019; Schneider et al., 2013; Willer et al., 2019). The benefits of early, active rehabilitation require early access to services that would support such an approach. In the New Zealand health context, concussion care is strongly influenced by ACC as the public insurance provider. Therefore, advocacy for policies that facilitate early access to physiotherapy is warranted. Given the management offered by physiotherapists (Figure 3), early access 
to physiotherapy services would promote an early and active approach to recovery from concussion.

A further challenge is the range of concussion services and providers, which creates significant challenges in continuity of care that may impact on outcomes. Responses highlight that people with concussion often access multiple services, and transitioning in and out of medical, community physiotherapy and specialist services, such as the ACC concussion service, can disrupt continuity of care. While this may be necessary to access relevant expertise, there is emerging evidence that early active rehabilitation may reduce delayed recovery and the need for specialist services (Leddy et al., 2019). Furthermore, while the ACC concussion service is fully funded, other services are not, creating inconsistencies in the cost of different services for individuals. An increased focus on early access to active rehabilitation services in acute concussion may be an effective use of health resources.

Many respondents $(120 ; 77.9 \%)$ indicated that at least "sometimes" people returned post discharge for further management of their concussion due to persistent problems. Types of problems identified related to multiple systems (Figure 4) and were sometimes multifactorial. The frequency of persistent symptoms is consistent with New Zealand research (Theadom et al., 2016), and highlights demand for access to physiotherapy services even after receiving an initial package of care. In knowing this, avenues for subsequent management of recurrent or persistent symptoms should be explored, as it is not clear how different services, especially contracted services, facilitate this. It is possible that the recovery rates and outcomes reported for sports-related concussion may differ from those for non-sports-related concussion (Leddy et al., 2012). Persistent problems are reported in only $10 \%$ of athletes with concussion (Leddy et al., 2012), while persistent problems in people with non-sports-related concussion recovery have been reported to be as high as $47.9 \%$ (Theadom et al., 2016). While multifactorial, this could in part relate to differences in access to health care, in particular access to early active rehabilitation (Leddy et al., 2016).

This study has limitations primarily related to its design. The response rate was limited to approximately $5 \%$ of PNZ members, and may not represent the views of the wider profession. The responses were considered broadly representative and suitable for the descriptive purposes of this study. An error in the survey logic led to the question in Table 3 not being displayed to some participants. Results were still considered reflective of the sample and have been presented. Responses are based on self-reported data from physiotherapists describing their involvement in and provision of services for people with concussion. Data are therefore not validated or verified, and represents the views of respondents.

\section{CONCLUSION}

This study describes physiotherapist involvement in the recognition, assessment, and management of concussion in New Zealand. The findings reflect that physiotherapists have a unique skill set in concussion care and are contributing to a wide range of services. The concussion care described by respondents strongly aligns with international research evidence, especially the trend towards early active rehabilitation in concussion. Challenges encountered by New Zealand physiotherapists include late presentation to physiotherapy, difficulty providing continuity of care through complex services, and managing people with persistent symptoms.

\section{KEY POINTS}

1. New Zealand physiotherapists are involved in a wide range of services for people with concussion. However, further work is needed to engage more of the profession in concussion care.

2. Physiotherapists have a unique skill set and describe care aligned with international recommendations, especially for early active rehabilitation and screening for concurrent injuries. Challenges highlighted include late presentation to physiotherapy, maintaining continuity of care through complex services, and managing people with persistent symptoms.

\section{DISCLOSURES}

No funding was received for this research. There are no conflicts of interest which may be perceived to interfere with or bias this study.

\section{PERMISSIONS}

Ethical approval was obtained from the University of Otago Human Ethics Committee (D19/187).

\section{ACKNOWLEDGEMENTS}

We would like to thank the many representatives of PNZ, including special interest groups and branches, who supported the distribution of the survey. We also thank all survey respondents for contributing to this work.

\section{ADDRESS FOR CORRESPONDENCE}

Dr Ewan Kennedy, School of Physiotherapy, University of Otago, Dunedin 9016, New Zealand.

Email: ewan.kennedy@otago.ac.nz

\section{REFERENCES}

Accident Compensation Corporation. (2016). Sport concussion in New Zealand: ACC national guidelines. https://www.healthnavigator.org.nz/ media/1001/acc-sportsmart-sport-concussion-in-new-zealand-acc-nationalguidelines.pdf

Elkin, B. S., Elliott, J. M., \& Siegmund, G. P. (2016). Whiplash injury or concussion? A possible biomechanical explanation for concussion symptoms in some individuals following a rear-end collision. Journal of Orthopaedic \& Sports Physical Therapy, 46(10), 874-885. https://doi. org/10.2519/jospt.2016.7049

Ellis, M. J., Leddy, J. J., \& Willer, B. (2015). Physiological, vestibulo-ocular and cervicogenic post-concussion disorders: An evidence-based classification system with directions for treatment. Brain Injury, 29(2), 238248. https:// doi.org/10.3109/02699052.2014.965207

Hsieh, H. F., \& Shannon, S. E. (2005). Three approaches to qualitative content analysis. Qualitative Health Research, 15(9), 1277-1288. https://doi. org/10.1177/1049732305276687

Kennedy, E., Quinn, D., Chapple, C., \& Tumilty, S. (2019). Can the neck contribute to persistent symptoms post concussion? A prospective descriptive case series. Journal of Orthopaedic \& Sports Physical Therapy, 49(11), 845-854. https://doi.org/10.2519/jospt.2019.8547 
Leddy, J. J., Baker, J. G., \& Willer, B. (2016). Active rehabilitation of concussion and post-concussion syndrome. Physical Medicine and Rehabilitation Clinics of North America, 27(2), 437-454. https://doi. org/10.1016/j.pmr.2015.12.003

Leddy, J. J., Haider, M. N., Ellis, M. J., Mannix, R., Darling, S. R., Freitas, M. S., Suffoletto, H. N., Leiter, J., Cordingley, D. M. \& Willer, B. (2019). Early subthreshold aerobic exercise for sport-related concussion: A randomized clinical trial. JAMA Pediatrics, 173(4), 319-325. https://doi.org/10.1001/ jamapediatrics.2018.4397

Leddy, J. J., Sandhu, H., Sodhi, V., Baker, J. G., \& Willer, B. (2012) Rehabilitation of concussion and post-concussion syndrome. Sports Health, 4(2), 147-154. https://doi.org/10.1177/1941738111433673

Leddy, J. J., Wilber, C. G., \& Willer, B. S. (2018). Active recovery from concussion. Current Opinion in Neurology, 31(6), 681-686. https://doi. org/10.1097NCO.0000000000000611

Leddy, J. J., \& Willer, B. (2013). Use of graded exercise testing in concussion and return-to-activity management. Current Sports Medicine Reports, 12(6), 370-376. https://doi.org/10.1249/JSR.0000000000000008

Leslie, O., \& Craton, N. (2013). Concussion: Purely a brain injury? Clinical Journal of Sport Medicine, 23(5), 331332. https://doi.org/10.1097/ JSM.0b013e318295bbb1

Marshall, S., Bayley, M., McCullagh, S., Velikonja, D., Berrigan, L. Ouchterlony, D., \& Weegar, K; mTBI Expert Consensus Group. (2015). Updated clinical practice guidelines for concussion/mild traumatic brain injury and persistent symptoms. Brain Injury, 29(6), 688-700. https://doi.or g/10.3109/02699052.2015.1004755

McCrory, P., Meeuwisse, W., Dvořák, J., Aubry, M., Bailes, J., Broglio, S., Cantu, R. C., Cassidy, D., Echemendia, R. J., Castellani, R. J., Davis, G. A., Ellenbogen, R., Emery, C., Engebretsen, L., Feddermann-Dermont, N., Giza, C. C., Guskiewicz, K. M., Herring, S., Iverson, G. L., . . Vos, P. E. (2017). Consensus statement on concussion in sport - the 5 th international conference on concussion in sport held in Berlin, October 2016. British Journal of Sports Medicine, 51(11), 838-847. https://doi. org/10.1136/bjsports-2017-097699

Physiotherapy Board of New Zealand. (2018). Annual report 2018. https://www.physioboard.org.nz/sites/default/files/ PhysiotherapyBoardAnnualReport2018.pdf

Physiotherapy New Zealand. (2019). Retrieved June 10, 2019, from https:// pnz.org.nz/

Reneker, J. C., Hassen, A., Phillips, R. S., Moughiman, M. C., Donaldson, M., \& Moughiman, J. (2017). Feasibility of early physical therapy for dizziness after a sports-related concussion: A randomized clinical trial. Scandinavian Journal of Medicine and Science in Sports, 27(12), 2009-2018. https://doi. org/10.1111/sms.12827
Schneider, D. K., Grandhi, R. K., Bansal, P., Kuntz, G. E., Webster, K. E., Logan, K., Barber Foss, K. D., \& Myer, G. D. (2017). Current state of concussion prevention strategies: A systematic review and meta-analysis of prospective, controlled studies. British Journal of Sports Medicine, 51(20) 1473-1482. https://doi.org/10.1136/bjsports-2015-095645

Schneider, K. J. (2019a). Concussion - Part I: The need for a multifaceted assessment. Musculoskeletal Science and Practice, 42, 140-150. https:// doi.org/10.1016/j.msksp.2019.05.007

Schneider, K. J. (2019b). Concussion part II: Rehabilitation - The need for a multifaceted approach. Musculoskeletal Science and Practice, 42, 151161. https://doi.org/10.1016/j.msksp.2019.01.006

Schneider, K. J., Iverson, G. L., Emery, C. A., McCrory, P., Herring, S. A., \& Meeuwisse, W. H. (2013). The effects of rest and treatment following sport-related concussion: A systematic review of the literature. British Journal of Sports Medicine, 47(5), 304-307. https://doi.org/10.1136/ bjsports-2013-092190

Schneider, K. J., Meeuwisse, W. H., Nettel-Aguirre, A., Barlow, K., Boyd, L., Kang, J., \& Emery, C. A. (2014). Cervicovestibular rehabilitation in sport-related concussion: A randomised controlled trial. British Journal of Sports Medicine, 48(17), 1294-1298. https://doi.org/10.1136/ bjsports-2013-093267

Theadom, A., Parag, V., Dowell, T., McPherson, K., Starkey, N., Barker-Collo, S., Jones, K., Ameratunga, S., \& Feigin, V. L; BIONIC Research Group. (2016). Persistent problems 1 year after mild traumatic brain injury: A longitudinal population study in New Zealand. British Journal of General Practice, 66(642), e16-23. https://doi.org/10.3399/bjgp16X683161

Vaismoradi, M., Jones, J., Turunen, H., \& Snelgrove, S. (2016). Theme development in qualitative content analysis and thematic analysis. Journal of Nursing Education and Practice, 6(5), 100-110. https://doi.org/10.5430/ jnep.v6n5p100

van der Walt, K., Tyson, A., \& Kennedy, E. (2019). How often is neck and vestibulo-ocular physiotherapy treatment recommended in people with persistent post-concussion symptoms? A retrospective analysis. Musculoskeletal Science and Practice, 39, 130-135. https://doi. org/10.1016/j.msksp.2018.12.004

Willer, B. S., Haider, M. N., Bezherano, I., Wilber, C. G., Mannix, R., Kozlowski, K., \& Leddy, J. J. (2019). Comparison of rest to aerobic exercise and placebo-like treatment of acute sport-related concussion in male and female adolescents. Archives of Physical Medicine and Rehabilitation, 100(12), 2267-2275. https://doi.org/10.1016/j.apmr.2019.07.003 